\section{Nanoparticles vs. bacteria}

\section{By Tim Fulmer, Senior Writer}

Researchers from Singapore and China have cobbled together multiple drug delivery and targeting strategies to create a single 20 -amino-acid peptide that self-assembles into an antimicrobial nanoparticle. ${ }^{1}$ The nanoparticles cross the blood brain barrier and have shown activity in rabbits infected with meningitis, although more studies are needed to show whether the compounds affect healthy tissue or provoke unwanted immune responses.

In designing the peptide, a group at Singapore's Institute of Bioengineering and Nanotechnology (IBN) and colleagues from Zhejiang University first had to figure out how to get it across the blood brain barrier (BBB). The solution was an 11-amino-acid domain of the HIV-1 tat protein.

Previous work had shown that the tat domain can mediate passage of proteins and nanoparticles across the endothelial cells of the BBB. ${ }^{2-4}$ And, although the domain is derived from a viral protein, it is not infectious.

The next step was getting antimicrobial activity. That function of the peptide was accomplished by adding a domain of six positively charged arginine residues. Positively charged peptides target and disrupt negatively charged cellular membranes, potentially killing both Gram-negative and Gram-positive bacteria as well as fungi and parasites. ${ }^{5}$

Finally, a hydrophobic block of cholesterol molecules was used to drive the self-assembly of the peptides into nanoparticles with a hydrophobic core and a positively charged peptide shell. Compared with free peptide, the peptide nanoparticles were expected to yield higher local concentrations of positively charged peptide at the bacterial membrane, thus enhancing antimicrobial activity.

The researchers designated the peptide nanoparticles $\mathrm{CG}_{3} \mathrm{R}_{6} \mathrm{TAT}$ to denote cholesterol (C), a spacer of three glycine residues $(\mathrm{G})$, the six arginine residues (R) and the tat domain (TAT).

In vitro, the nanoparticles showed broad-spectrum activity against a variety of bacteria, yeast and fungi, including Staphylococcus aureus, methicillin-resistant S. aureus (MRSA), vancomycinresistant Enterococcus (VRE) and Candida albicans. Minimal inhibitory concentrations (MICs) typically were $2-12 \mu \mathrm{M}$.

The nanoparticles were then intravenously delivered to a rabbit model of $S$. aureus-induced meningitis to see if they could cross the $\mathrm{BBB}$ and treat the brain infection.
Indeed, brain tissue isolated from the rabbits 36 hours following treatment had lower levels of $S$. aureus than brain tissue from rabbits that received vehicle control. Nanoparticle-treated rabbits had levels of $S$. aureus that were comparable to those in rabbits treated with vancomycin.

Neutrophil levels in both the cerebrospinal fluid and peripheral blood were lower in rabbits that received the nanoparticles than in vehicle-treated animals, suggesting a lower rate of infection.

At low doses, the nanoparticles induced less hemolysis of red blood cells than did amphotericin B ( $20 \%$ vs. $90 \%$ ), a generic antibiotic and antifungal that is often used to treat meningitis. Also, serum analysis of the meningitis-infected rabbits that received the nanoparticles showed no elevation of key liver enzymes and renal metabolites compared with what was seen in untreated controls.

The findings were published in Nature Nanotechnology.

\section{Resolving immunogenicity}

"The paper shows that the peptide nanoparticles can disrupt bacterial cell membranes and kill bacteria. However, further studies are necessary to confirm that the peptides have sufficient selectivity for bacteria, because the findings also showed some disruption of the membranes of red blood cells, which have a known susceptibility to hemolysis by cationic peptides," said John North, president and $\mathrm{CEO}$ of Inimex Pharmaceuticals Inc.

Inimex is developing innate defense regulator (IDR) peptides designed to enhance the host's innate immune response to infection rather than directly targeting bacteria. IMX942, the company's lead compound, is in Phase I safety testing in healthy volunteers, with plans to begin a Phase II trial to treat hospital bacterial infections in 1Q10, said North.

It will also be important to rule out any potential immunogenicity associated with the peptide nanoparticles, according to Richard Scott, VP of research at PolyMedix Inc., which develops small molecule mimetics of antimicrobial peptides.

North suggested a set of animal experiments that could help clarify the immunogenicity issue.

"Long-term monitoring of serum antibodies and a skin sensitivity test, as well as levels of proinflammatory cytokines like TNF [tumor necrosis factor], could help show if the nanoparticles are immunogenic or trigger significant inflammation," he told SciBX. "Postmortem analysis of lung, liver and spleen tissue would also reveal whether those organs clear large numbers of the nanoparticles from circulation before they reach infected tissue and thus lower their potential antimicrobial activity."

The peptide nanoparticles are being characterized in preclinical models of undisclosed infectious diseases to determine whether they induce undesired host immune responses, said Yi-Yan Yang, corresponding author and IBN group leader.

Nonetheless, an ongoing concern over immunogenicity is one of 
the reasons some biotechs opt to develop small molecule mimetics of antibacterial peptides rather than the peptides themselves, said Scott.

A case in point is Ceragenix Pharmaceuticals Inc. It is developing small molecule cationic steroid antibiotics that bind bacterial membranes and induce apoptosis in a manner somewhat similar to the peptide described in the Nature Nanotechnology paper, according to chairman and CEO Steven Porter.

He noted other advantages of small molecule peptide mimetics include ease of manufacture and improved stability compared with peptides. Ceragenix's lead compound, CSA-13, has shown activity against Gram-positive and Gram-negative bacteria, Candida fungi and orthopoxviruses in preclinical models.

PolyMedix has also opted for the peptide mimetics route. PMX30063, a small molecule mimic of host defense protein defensin, is in Phase Ib testing to treat drug-resistant and drug-sensitive staphylococcal infection.

\section{Reducing cost}

Once the toxicity profile of the nanoparticles has been fleshed out, another step could include optimizing the length of the nanoparticle peptides.

"As it is, the 20-amino-acid peptide presented in the paper would probably be quite complex and rather costly to manufacture. It will therefore definitely be worth finding out if the peptide can be shortened without impairing its in vivo antimicrobial activity," said Bob Hancock, cofounder of Inimex, director of the Centre for Microbial Diseases and Immunity Research and professor of microbiology and immunology at The University of British Columbia.

Hancock and colleagues have developed nine-amino-acid peptides that have shown broad-spectrum antimicrobial activity in vitro and in mouse models of $S$. aureus infection. ${ }^{6}$

At least one other lab has designed shorter antimicrobial peptides. M. Reza Ghadiri and colleagues at The Scripps Research Institute created eight-amino-acid cyclic peptides that assembled into nanotubes and showed antibacterial activity in mice infected with MRSA. ${ }^{7}$

Yang told SciBX she hopes to test the peptide nanoparticles in clinical trials. The researchers have applied for a patent covering the peptide nanoparticle technology, and the IP is available for licensing.

Fulmer, T. SciBX 2(29); doi:10.1038/scibx.2009.1130

Published online July 30, 2009

\section{REFERENCES}

1. Liu, L. et al. Nat. Nanotechnol.; published online June 28, 2009; doi:10.1038/NNANO.2009.153

Contact: Yi-Yan Yang, Institute of Bioengineering and Nanotechnology, Singapore

e-mail: yyyang@ibn.a-star.edu.sg

Contact: Lanjuan Li, Zhejiang University, Hangzhou, China e-mail: ljli@zju.edu.cn

2. Liu, L. et al. Biomaterials 29, 1509-1517 (2008)

3. Santra, S. et al. Chem. Commun. (Camb.) 25, 3144-3146 (2005)

4. Chauhan, A. et al. J. Control. Release 117, 148-162 (2007)

5. Hancock, R. \& Sahl, H.-G. Nat. Biotechnol. 24, 1551-1557 (2006)

6. Cherkasov, A. et al. ACS Chem. Biol. 4, 65-74 (2008)

7. Fernandez-Lopez, S. et al. Nature 412, 452-455 (2001)

\section{COMPANIES AND INSTITUTIONS MENTIONED}

Ceragenix Pharmaceuticals Inc. (OTCBB:CGXP), Denver, Colo. Inimex Pharmaceuticals Inc., Vancouver, British Columbia, Canada Institute of Bioengineering and Nanotechnology, Singapore

PolyMedix Inc. (OTCBB:PYMX), Radnor, Pa.

The Scripps Research Institute, La Jolla, Calif.

The University of British Columbia, Vancouver, British Columbia, Canada Zhejiang University, Hangzhou, China 